\title{
Three-dimensional simulation study of magnetic reconnection in the solar corona
}

\author{
Kanya Kusano \\ Graduate School of Advanced Sciences of Matter, Hiroshima University, Higashi-Hiroshima 739-8526, Japan
}

(Received October 2, 2000; Revised December 11, 2000; Accepted December 12, 2000)

\begin{abstract}
A three-dimensional reconnection process, which is caused by the magnetic arcade instability, is investigated using numerical simulations. It is found that the aspect ratio (defined as the ratio of the length to the width of the magnetic arcade) is a crucial parameter to control the nonlinear dynamics. When the aspect ratio is rather small, the dynamics is governed by a symmetric mode, and more explosive reconnection happens. In a longer arcade, on the other hand, an undulating three-dimensional activity arises. It suggests that the variety in solar coronal activity may be caused by the differece in arcade shape.
\end{abstract}

\section{Introduction}

Magnetic arcades are formed in elongated bipolar regions on the solar surface, and are widely believed to be responsible for several sorts of solar coronal activity, especially in two ribbon flares. Actually, X-ray observations by Yohkoh and $T R A C E$ evidently indicate that some flares are brought about by magnetic reconnection in magnetic arcades (Tsuneta, 1996; Warren et al., 1999).

Due to the elongated structure in magnetic arcades, most of the previous flare models have been developed in twodimensional (2D) geometry, where any dependency along the neutral line is neglected (Mikic et al., 1988; Biskamp and Welter, 1989; Mikic and Linker, 1994). However, since the practical system can evolve in three-dimensional (3D) space, it is crucially important to understand the 3D effects of the reconnection process in magnetic arcades (Priest and Forbes, 2000), and the numerical models for 3D dynamics is being developed recently (Kusano et al., 1994; Suzuki et al., 1997; Amari et al., 2000).

One of the most important 3D effects is the 3D mode instability, which spontaneously breaks the symmetry in the magnetic arcades. The primary objective in this paper is to investigate the reconnection dynamics driven by the $3 \mathrm{D}$ instability in the solar coronal magnetic arcades using the magnetohydrodynamic (MHD) numerical simulations. Particularly, we aim to reveal the relationship between reconnection dynamics and the arcade geometry.

When we consider the 3D instability, the minimum energy theory (Taylor, 1986), which predicts that the minimum energy state is given by the linear force-free field, $\nabla \times \boldsymbol{B}=\alpha \boldsymbol{B}$, may work as a guiding principle. Particularly, Kusano et al. (1995) found that the minimum energy state in periodic magnetic arcades bifurcates between an arcade-type field and a plasmoid-type field, if $\alpha$ is larger than the threshold. Once the bifurcation happens, the minimum energy state is the plasmoid-type solution instead of the arcade-type

Copy right (C) The Society of Geomagnetism and Earth, Planetary and Space Sciences (SGEPSS); The Seismological Society of Japan; The Volcanological Society of Japan; The Geodetic Society of Japan; The Japanese Society for Planetary Sciences. solution. This indicates that magnetic reconnection could spontaneously take place when the magnetic helicity in the coronal field is sufficiently large.

Actually, the linear stability analysis shows that the arcadetype solution is destabilized against the so-called magnetic arcade instability (MAI), when $\alpha$ is larger than the bifurcation threshold. Furthermore, the bifurcation theory predicts that not only the $2 \mathrm{D}$ mode but also the $3 \mathrm{D}$ mode, which undulates the arcade along the magnetic neutral line, becomes unstable (Kusano and Nishikawa, 1996). Here, in this study, we attempt to examine whether the 3D instability may grow in a magnetic arcade subjected to dynamical helicity injection.

Recently, Kusano (1999) calculated also the long-term dynamics of $2 \mathrm{D}$ magnetic arcades that are subjected to constant helicity injection, and showed that a cyclic activity proceeds in the case that the magnetic Reynolds number is high enough. Each cycle consists of the following three phases. The first phase is a pumping phase, in which the magnetic helicity is stored in the arcades. The second phase is a relaxation phase, in which the MAI is destabilized and reconnection takes place. The final phase is a recovery phase, in which the reconnection ejects a plasmoid and the stability of the magnetic arcade is recovered. The study of long-term dynamics in the 3D system is another subject in this paper, particularly aiming to clarify how different is the 3D dynamics from the $2 \mathrm{D}$ case.

\section{Numerical Model}

The simulation domain is a rectangular box, which spans the region $0<x<L_{x}, 0<y<L_{y}$ and $0<z<L_{z}$ in the Cartesian coordinate $(x, y, z)$. Here, the inside of the box is the coronal region, and the bottom plane $(z=0)$ corresponds to the photospheric surface. The basic equations to be solved consist of the equation of motion and the induction equation, where the plasma pressure and the gravity force are omitted, as in previous studies (Mikic et al., 1988; Kusano, 1999). The viscosity and the resistivity are constant in space and time, and the Reynolds number as well as the magnetic Reynolds 


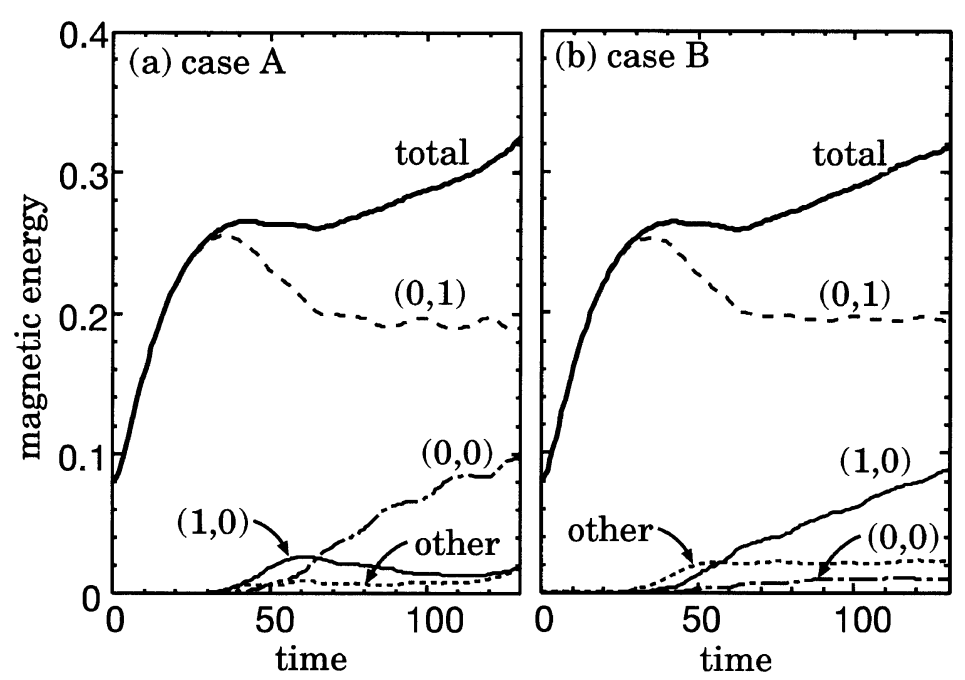

Fig. 1. The temporal evolution of the magnetic energy for several major Fourier components, $(m, n)=(0,0),(0,1)$ and $(1,0)$, is plotted (a) for case A $\left(L_{x} / L_{y}=5\right)$, and (b) case B $\left(L_{x} / L_{y}=10\right)$, respectively.
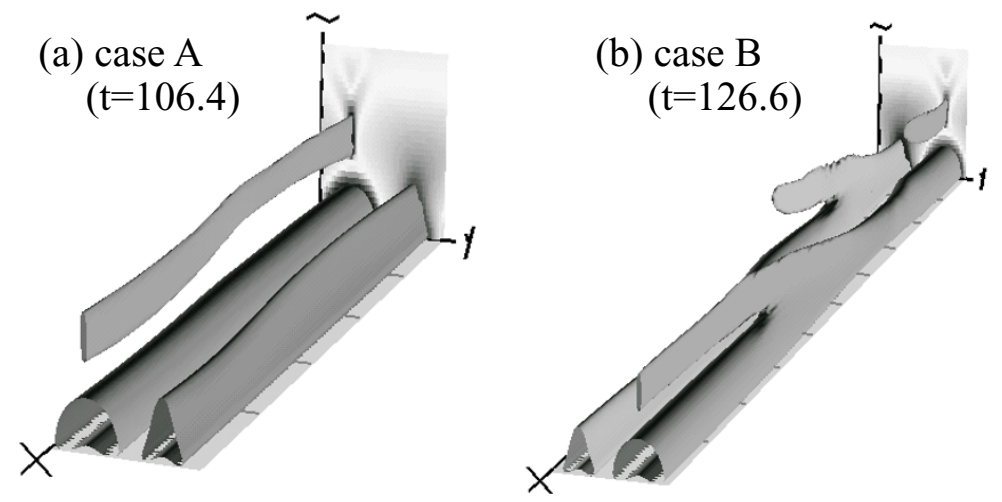

Fig. 2. Three dimensional snap shots of the iso-surface for the electric current density $\left|J_{x}\right|=2.5$ are drawn (a) for case A and (b) for case B.

number, those are normalized by the Alfvén speed and the domain size $\left(L_{y}\right)$, are $10^{3}$.

The boundary condition employed here is also similar to previous papers (Mikic et al., 1988; Kusano, 1999). On the lateral boundaries, the periodic condition is applied, and on the bottom boundary the normal magnetic field and the velocity vector are specified so that $B_{z}=B_{0} \cos \left(2 \pi y / L_{y}\right)$, $V_{x}=V_{0} \sin \left(4 \pi y / L_{y}\right)$ and $V_{y}=V_{z}=0$. If we expand the boundary condition by a $2 \mathrm{D}$ complex Fourier series such that $f(x, y, z)=\sum_{m, n} \tilde{f}_{m, n}(x) \exp \left[i 2 \pi\left(\frac{m x}{L_{x}}+\frac{n y}{L_{y}}\right)\right]$, the magnetic field $B_{z}$ and the velocity $V_{x}$ on the bottom boundary, respectively, have just a single Fourier mode; $(m, n)=(0,1)$ for $B_{z}$ and $(0,2)$ for $V_{x}$. Therefore, also the Poynting flux across the bottom boundary possesses only the $m=0$ modes. It implies that, if the $m \neq 0$ components grow, it must be the result of a 3D instability. The initial state is composed of the potential field, which satisfies $\nabla \times \boldsymbol{B}=0$, plus a small perturbation, which is necessary as the seed for the instability.

The numerical solver consists of a second-order accurate finite difference and a pseudo-spectral method based on the FFT technique. The latter is used to approximate the differential along the arcade $(\partial / \partial x)$. The grid number for the $y$ and $z$ coordinates $\left(N_{y}\right.$ and $\left.N_{z}\right)$ are 128 and 256, where the grid size is spatially modulated in order to accurately capture the thin current layers on the reconnection site. The Fourier modes included in the model are $m=-32$ to 32 , in which the high modes $|m|>21$ are filtered out each time step to prevent the aliasing error. The time integration is carried out using the Runge-Kutta-Gill method.

We mainly study the geometrical effect on the reconnection dynamics by calculating three different cases (A, B and C), those representing a short, a long, and a very long arcade, where $L_{x} / L_{y}=5,10$, and 20, respectively.

\section{Simulation Results}

Figure 1 shows the magnetic energy evolution for the major Fourier modes. It is seen that the main arcade component, $(m, n)=(0,1)$, behaves similarly in either case. The initial pumping up phase $(t=0-40)$ is followed by the relaxation phase $(t=40-60)$, and then the quasi-steady process is obtained, where it stays in a level about 0.2 . The quasisteady process consists of cyclic energy relaxation, in which the magnetic arcade is periodically destabilized and transfers the free energy to other modes, as in the $2 \mathrm{D}$ dynamics 
(a) case A

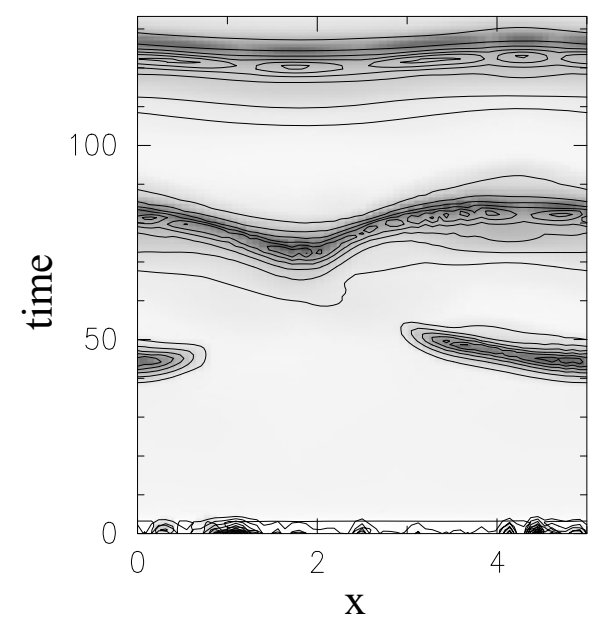

(b) case B

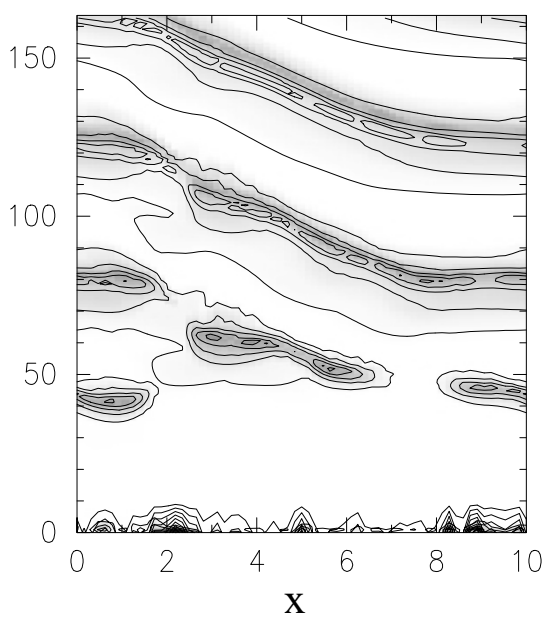

(c) case $\mathrm{C}$

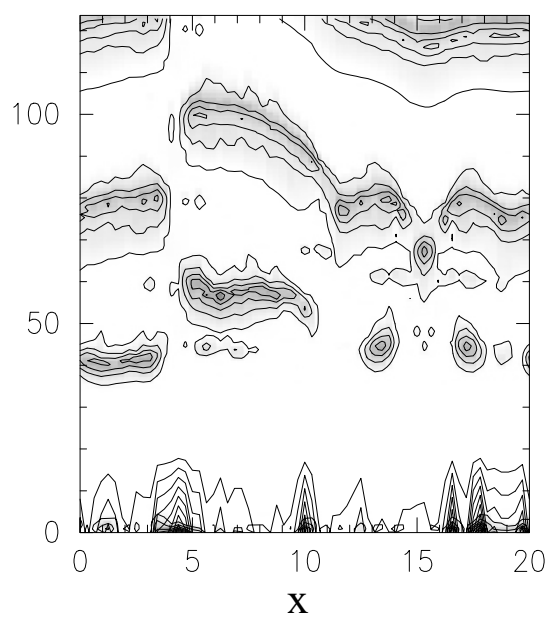

Fig. 3. The spatio-temporal structure of the peaked current intensity is plotted on the $x$ vs. $t$ plane. The gray scale indicates the maximum current density in a cross section $(x=$ const.), and the darker region represents the evolution of the intense current sheets.

(a)

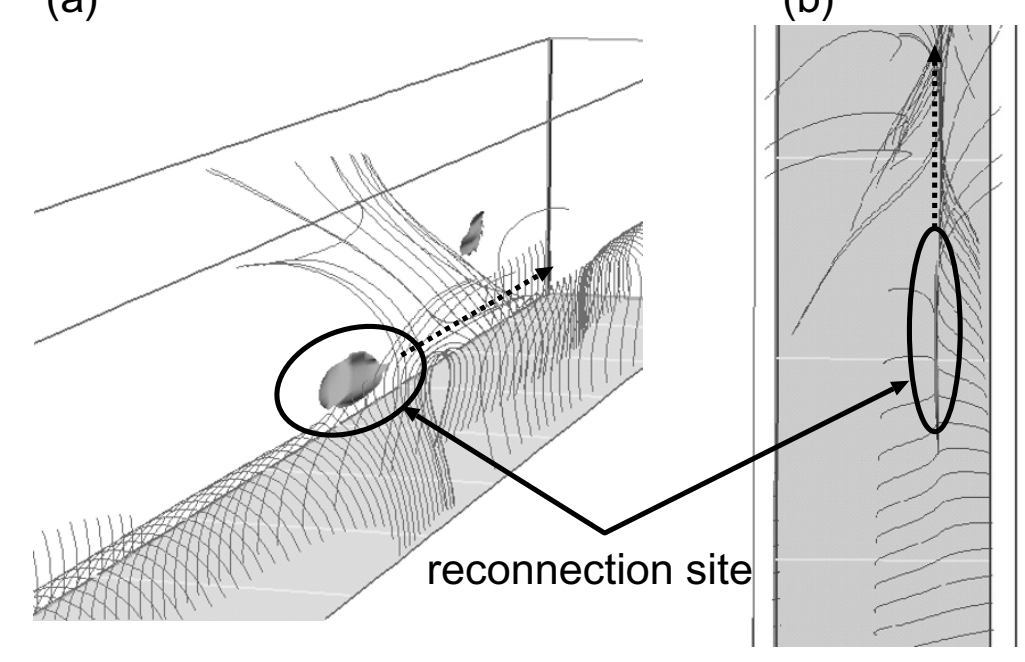

Fig. 4. The magnetic field lines of force as well as the reconnection site, which corresponds to the strong current region, are drawn, where (a) and (b) show the side view and the top view for case B. The dotted arrow indicates the traveling direction of the reconnection site.

(Kusano, 1999). However, notice that the growing mode in the cyclic relaxation process is different between cases $\mathrm{A}$ and $\mathrm{B}$. The symmetric mode $(0,0)$ grows in case $\mathrm{A}$, but the undulating mode $(1,0)$ does in case $\mathrm{B}$. It means that the $3 \mathrm{D}$ instability arises in the long arcade system, while quasi-2D activity proceeds in the relatively compact arcade. It is also observed in the cyclic relaxation phase that the amplitude in each energy relaxation of the $(0,1)$ mode for case $A$ is about five times larger than that for case B.

The structural difference between the quasi-2D and 3D dynamics can be seen in Fig. 2, which shows the 3D shape of the current sheet. In case A (Fig. 2(a)), the current sheet is almost uniformly distributed along the magnetic arcade, whereas in case B (Fig. 2(b)) it appears only on a limited region of the arcade. It means that magnetic reconnection in the long arcade proceeds in a restricted region, while all field lines are reconnected simultaneously in the compact arcade. The difference in the oscillating amplitude of the $(0,1)$ mode between cases A and B can be explained by the size effect of the reconnection region. These results suggest that the length of the reconnection region could be limited by spontaneous dynamics when the magnetic arcade is long enough.

Figure 3 indicates the spatio-temporal structure of the current sheets, showing how the reconnection site evolves along the arcade. Note that the current sheet appearing first at $t \sim 40$ is not spread over the whole domain in all cases, indicating the 3D structure. In case A, however, the dependency on $x$ is weakened in the following process, and the current sheet generated later behaves quasi-two dimensionally. On the other hand, it is seen that the current sheet in the long arcade system (case B) travels to the anti- $x$ direction, not spreading along the arcade axis. The traveling of the current 
sheet might be a natural result in the localized reconnection process, because the reconnection point must move around the domain in order to release the free energy uniformly supplied into the whole domain from the boundary. Actually, Fig. 4 clearly shows that the magnetic shear is relaxed after the reconnection site passes through.

In case $C$, where the arcade is more elongated $\left(L_{x}=20\right)$, the current sheet is fragmented into several portions as seen in Fig. 3(c), and each fragmented current sheet evolves in a complicated manner, which seems to be mixture of the traveling process observed in case $\mathrm{B}$ and the standing oscillation appearing in case A. Here, we may conclude that the length of the arcade could be a key parameter for 3D reconnection activity, and that the long arcade, where the aspect ratio is larger than about ten, may evolve three-dimensionally. On the other hand, in the compact arcade a coherent reconnection may release the free energy more explosively.

McKenzie and Hudson (1999) observed a long-duration event in 1999 January 20 with the Yohkoh soft X-ray telescope (SXT; Tsuneta et al., 1991), and found that several blobs intermittently moved downward in the arcade fan, which presumably connects to the current sheet above the arcade. The most likely scenario to explain this is that the moving blobs are the downward outflow ejected by $3 \mathrm{D}$ reconnection. It is similar to the complicated evolution of the fragmented current sheets observed in the very long arcade simulation.

\section{Summary}

We have studied the nonlinear evolution driven by the magnetic arcade instability. The simulations indicate that $3 \mathrm{D}$ reconnection takes place when the arcade length is longer than some critical value. The simulation also shows that the energy release with the $3 \mathrm{D}$ reconnection is moderate compared to the quasi-2D reconnection which happens in the compact arcade. It could be because the $3 \mathrm{D}$ system has more freedom of the dynamics than the $2 \mathrm{D}$ system. In the $2 \mathrm{D}$ and the quasi-2D systems, since the dynamical path to access the lower-energy state is more restricted than the $3 \mathrm{D}$ system, a larger free energy has to be stored to drive the instability, and more explosive energy liberation may result.

Finally, it should be mentioned that the boundary condition employed here is much idealized compared to the practical solar surface, because we focus here on the theoretical study of the 3D instability. Hence, further study is necessary to examine more realistic dynamics.

Acknowledgments. The author is grateful to the Japan Society for the Promotion of Science for the financial support by a Grant-in-Aid for Scientific Research.

\section{References}

Amari, T., J. F. Luciani, Z. Mikic, and J. Linker, A twisted flux rope model for coronal mass ejections and two-ribbon flares, Astophys. J., 529, L49L52, 2000.

Biskamp, D. and H. Welter, Magnetic arcade evolution and instability, Solar Phys., 120, 49-77, 1989.

McKenzie, D. E. and H. S. Hudson, X-Ray observations of motions and structure above a solar flare arcade, Astrophys. J., 519, L93-L96, 1999.

Mikic, Z. and J. A. Linker, Disruption of coronal magnetic field arcades, Astrophys. J., 430, 898-912, 1994.

Mikic, Z., D. C. Barnes, and D. D. Schnack, Dynamical evolution of a solar coronal magnetic field arcade, Astrophys. J., 328, 830-847, 1988.

Kusano, K., Magnetic helicity and stability in solar corona, in Geophysical Monograph 111, Magnetic Helicity in Space and Laboratory Plasmas, edited by M. R. Brown, R. C. Canfield, and A. A. Pevtsov, pp. 149-156, Washington D.C., AGU, 1999.

Kusano, K. and K. Nishikawa, Bifurcation and stability of coronal magnetic arcades in a linear force-free field, Astrophys. J., 461, 415-423, 1996.

Kusano, K., Y. Suzuki, H. Kubo, T. Miyoshi, and K. Nishikawa, Threedimensional simulation study of the magnetohydrodynamic relaxation process in the solar corona. 1: Spontaneous generation of TaylorHeyvaerts-Priest state, Astrophys. J., 433, 361-378, 1994.

Kusano, K., Y. Suzuki, and K. Nishikawa, A solar flare triggering mechanism based on the Woltjer-Taylor minimum energy principle, Astrophys. J., 441, 942-951, 1995.

Priest, E. R. and T. G. Forbes, Magnetic Reconnection, 600 pp., Cambridge University Press, Cambridge, 2000.

Suzuki, Y., K. Kusano, and K. Nisikawa, Three-dimensional simulation study of the magnetohydrodynamic relaxation process in the solar corona. II, Astrophys. J., 474, 782-789, 1997.

Taylor, J. B., Relaxation and magnetic reconnection in plasmas, Rev. Mod. Phys., 58, 741-763, 1986.

Tsuneta, S., Structure and dynamics of magnetic reconnection in a solar flare, Astrophys. J., 456, 840-849, 1996.

Tsuneta, S., et al., The soft X-ray telescope for the SOLAR-A mission, Solar Phys., 136, 37-67, 1991

Warren, H. P., J. A. Bookbinder, T. G. Forbes, L. Golub, H. S. Hudson, K. Reeves, and A. Warshall, TRACE and Yohkoh observations of hightemperature plasma in a two-ribbon limb flare, Astrophys. J., 525, L121L124, 1999.

K. Kusano (e-mail: kusano@sci.hiroshima-u.ac.jp) 